\title{
COLUMN SUMS AND THE CONDITIONING OF THE STATIONARY DISTRIBUTION FOR A STOCHASTIC MATRIX
}

\author{
STEVE KIRKLAND
}

\begin{abstract}
For an irreducible stochastic matrix $T$, we consider a certain condition number $\kappa(T)$, which measures the sensitivity of the stationary distribution vector to perturbations in $T$, and study the extent to which the column sum vector for $T$ provides information on $\kappa(T)$. Specifically, if $c^{T}$ is the column sum vector for some stochastic matrix of order $n$, we define the set $\mathscr{S}(c)=\left\{A \mid A\right.$ is an $n \times n$ stochastic matrix with column sum vector $\left.c^{T}\right\}$. We then characterise those vectors $c^{T}$ such that $\kappa(T)$ is bounded as $T$ ranges over the irreducible matrices in $\mathscr{S}(c)$; for those column sum vectors $c^{T}$ for which $\kappa$ is bounded, we give an upper bound on $\kappa$ in terms of the entries in $c^{T}$, and characterise the equality case.
\end{abstract}

Mathematics subject classification (2010): 15A51, 60J10, 65F35.

Keywords and phrases: Stochastic matrix, Stationary distribution, Condition number.

\section{REFERENCES}

[1] S. Campbell And C. Meyer, Generalized Inverses of Linear Transformations, Dover, New York, 1991.

[2] G. Cho AND C. Meyer, Comparison of perturbation bounds for the stationary distribution of a Markov chain, Linear Algebra and its Applications, 335 (2001), 137-150.

[3] M. HAVIV AND L. VAN DER HEYDEn, Perturbation bounds for the stationary probabilities of a finite Markov chain, Advances in Applied Probability, 16 (1984), 804-818.

[4] S. KIRKLAND, On a question concerning condition numbers for Markov chains, SIAM Journal on Matrix Analysis and Applications, 23 (2002), 1109-1119.

[5] S. KIRKLAND, Conditioning properties of the stationary distribution for a Markov chain, Electronic Journal of Linear Algebra, 10 (2003), 1-15.

[6] S. KIRKLAND, A combinatorial approach to the conditioning of a single entry in the stationary distribution for a Markov chain, Electronic Journal of Linear Algebra, 11 (2004), 168-179.

[7] S. KIRKLAND, Digraph-based conditioning for Markov chains, Linear Algebra and its Applications, 385 (2004), 81-93.

[8] S. KiRKLAND, Conditioning of the entries in the stationary vector of a Google-type matrix, Linear Algebra and its Applications, 418 (2006), 665-681.

[9] S. Kirkland, M. Neumann And B. Shader, Applications of Paz's inequality to perturbation bounds for Markov chains, Linear Algebra and its Applications, 268 (1998), 183-196.

[10] C. MEYER, The condition of a finite Markov chain and perturbation bounds for the limiting probabilities, SIAM Journal on Discrete and Algebraic Methods, 1 (1980), 273-283.

[11] E. SEnetA, Non-negative Matrices and Markov Chains, Springer-Verlag, New York, 1981. 